\title{
Diffuse Supernova Neutrino Background search at Super-Kamiokande with neutron tagging
}

\section{Alberto Giampaolo ${ }^{a, *}$ and Sonia El Hedri ${ }^{a}$ on behalf of the Super-Kamiokande Collaboration}

(a complete list of authors can be found at the end of the proceedings)

${ }^{a}$ École Polytechnique, IN2P3-CNRS, Laboratoire Leprince-Ringuet

Route de Saclay, 91120 Palaiseau, France

E-mail: giampaolo@llr.in2p3.fr, elhedri@llr.in2p3.fr

Detecting the Diffuse Supernova Neutrino Background at Super-Kamiokande requires designing state-of-the-art background removal technique to reject radioactivity induced by cosmic muon spallation, and identify atmospheric neutrino interactions. Identifying the neutron produced by the interaction of DSNB antineutrinos would allow to remove most of these backgrounds, but is particularly challenging in pure water. With the advent of the SK-Gd era, with Gadolinium being dissolved in the SK water, the efficiency of the neutron tagging procedure will increase dramatically, and the SK experiment will make significant gains in its sensitivity to the DSNB. I will present the role of neutron tagging and the challenges it provides, as well as discuss the impact of the SK-Gd project.

$37^{\text {th }}$ International Cosmic Ray Conference (ICRC 2021)

July 12th-23rd, 2021

Online - Berlin, Germany

\footnotetext{
*Presenter
} 


\section{The Diffuse Supernova Neutrino Background}

Core-collapse supernovae, dramatic explosions typically brighter than their host galaxy, mark the death of massive stars, and are characterized by a violent shockwave whereby more than $99 \%$ of the binding energy is released in the form of neutrinos. The Diffuse Supernova Neutrino Background is the flux of neutrinos and antineutrinos from all causally reachable core-collapse supernovae. As its name suggests, the DSNB will appear isotropic and time-independent for practical observations. Expectations for the DSNB flux can be estimated by convolving the neutrino energy spectrum of newborn neutron stars, in the $\mathrm{MeV}$ range, with the cosmic supernova rate. The DSNB energy density, $\sim 0.01 \mathrm{eV} / \mathrm{cm}^{-3}$ is about one tenth of that of the cosmic microwave background, and is comparable to that of photon emission from stars [1].

Motivations for the detection of the DSNB include the furthering of our understanding of cosmological evolution, of core-collapse supernovae, and of the properties of neutrinos themselves. Detection of the DSNB flux and its eventual characterization would be able to provide an independent measurement for the rate of stellar formation, while providing a wealth of information about black hole formation, chemical element synthesis, and matter effects of the neutrinos themselves, among other physical interests. Since, unlike photons, neutrinos quickly travel through the core itself and the stellar envelope undisturbed after their emission, they are a clean probe of the physics in the core and provide complementary information to optical detection of supernovae.

The interest in looking for an integrated supernova flux is that single "nearby" supernova bursts from within the Milky Way, which would provide strong enough neutrino signals, are very rare, with a galactic core-collapse supernova rate of only about 1-3 per century (only one supernova has so far been observed by neutrino observatories, SN1987A). By contrast, the DSNB is a guaranteed steady source. The chance of detecting neutrino emissions from a given supernova is extraordinarily low, but this is counterbalanced by an abundant cosmic burst rate, of about 10/second in the visible universe. While the DSNB has so not yet been detected, current experimental setups make discovery a possibility in the near future.

\subsection{Detection with the Super-Kamiokande Detector}

Super-Kamiokande (SK) [2] is a water Cherenkov detector located within the Kamioka mine, under Mt. Ikeno, in Japan's Gifu prefecture. Being $1 \mathrm{~km}$ high, the mountain provides shielding against cosmic muons, reducing their incidence by 5 orders of magnitude. The detector consists of a cylindrical stainless steel tank, $39.3 \mathrm{~min}$ diameter and $41.3 \mathrm{~m}$ tall, containing $50 \mathrm{kton}$ of ultrapure water. A stainless steel structure subdivides the detector into the cylindrical Inner Detector, and the surrounding Outer Detector, whose purpose is to monitor and veto cosmic muon events. SK is well-suited for a wide range of analyses: in addition to the DSNB, the detector is used to study solar and atmospheric neutrinos, search for proton decay, as well as keep watch for supernova bursts from inside the Milky Way and study neutrino oscillations by acting as the far detector to the longbaseline T2K experiment. Neutrino interactions in water are detected within the Inner Detector volume through detection of Cherenkov radiation from charged leptons or hadrons participating in the interaction. The particle's energy can be reconstructed from the number of Cherenkov photons registered in one of SK's 11,146 $51 \mathrm{~cm}$-wide Photomultiplier tubes (PMTs) arranged in the walls of the inner detector, which cover about $40 \%$ of the total photon production. 
SK's fiducial volume of $22.5 \mathrm{ktons}$ is is large enough for us to expect $\sim 3-10$ DSNB events per year. The detection channel is through the inverse beta decay (IBD) on protons in pure water:

$$
\bar{v}_{e}+\mathrm{p} \rightarrow e^{+}+\mathrm{n}
$$

To achieve the most stringent background rejection, it is crucial to identify both positron and neutron in coincidence. In particular, after the prompt Cherenkov signal from the $\mathrm{e}^{+}$, the thermal neutron is captured by one of the protons in the water, with emission of a $2.223 \mathrm{MeV}$ photon:

$$
\mathrm{n}+\mathrm{p} \rightarrow d+\gamma(2.223 \mathrm{MeV})
$$

The corresponding Cherenkov light signal, when it exists, is extremely faint and difficult to distinguish from dark noise. In addition, the neutron capture time in water is of $\tau_{\text {cap }}=204.8 \mu \mathrm{s}$, about 1000 times larger than the characteristic time scale of a single SK event. Tagging neutrons therefore requires exploring a time window significantly larger than the one typically used for SK triggers, and requires a particularly accurate modeling of the noise in SK.

To improve the chances of detecting neutron captures in the tank, Gadolinium sulfate has been dissolved in the detector's water in the summer of 2020 [3]. Gd boasts a much higher neutron capture cross-section than Hydrogen, and the signature of a neutron capture on $\mathrm{Gd}$ is a photon cascade with a total energy of $\sim 8 \mathrm{MeV}$, much brighter than in pure water. Here, we describe the neutron tagging procedure used for the DSNB search using the SK-IV data-taking period (Sep. 2008 - May 2018) and its role in the analysis. Secondly, we discuss the impact of neutron tagging for future DSNB searches with Gd.

\section{Neutron tagging in pure water}

Because of the large characteristic neutron capture time in water, the neutron search is carried out using the a dedicated trigger system spanning $535 \mu$ s after the primary event. This trigger system was developed for SK-IV, so this it is the first data-taking period at SK to make use of neutron tagging in a DSNB search [4]. Since the neutron capture signal is extremely weak the neutron tagging algorithm is highly sensitive to the noise from PMTs and radioactive decays in SK. Instead of simulating such backgrounds, we therefore estimate them directly from data, using samples of continuous data collected over the SK-IV period, triggered at random times (thus uncorrelated with a primary event). We inject the hits from this background data into MC simulations of IBD signal and of irreducible atmospheric background.

\subsection{Neutron candidate selection}

After filtering each event by removing noise-like and muon-like events, we are still left with $\sim 20000$ hits, much larger than for a neutron capture generally yielding $<10$ hits. The key advantage in such a search is that thermal neutrons travel at most a few tens of centimeters before being captured, in the order of the spatial resolution of SK $(\sim 50 \mathrm{~cm})$. Therefore, though the neutron capture vertex cannot be directly reconstructed, we can, to a good approximation, use the wellreconstructed $\mathrm{e}^{+}$production vertex instead to subtract time-of-flight from the hit timings, allowing 
to us to cluster $90 \%$ of the neutron hits inside a 10 ns window. A neutron candidate is defined as a cluster of hits in this window verifying

$$
N_{10}>5 \text {, }
$$

with $\mathrm{N}_{10}$ being the number of hits in the 10-ns window. Due to the weakness of the neutron signal, this preselection procedure has an efficiency of $45 \%$. In addition, a typical primary event, will contain about 11 candidates due to accidental coincidence from background.

\subsection{Boosted decision tree classification}

For the purposes of our search, our neutron identification algorithm needs to have background rates as low as $10^{-3}$. We therefore need to identify a large array of relevant observables and fully exploit their possible correlations. An ideal tool for this type of analysis is a Boosted Decision Tree (BDT), a machine learning method. For each neutron candidate, i.e. a cluster of hits in a 10 ns time-of-flight subtracted window, we consider 22 observables that can be grouped into three categories:

Neutron vertex fit In addition to the $N_{10}$ variable, we use the time-minus-time-of-flight spread of the candidate hits, $t_{\mathrm{rms}}$, as well as variables related to direct neutron capture vertex reconstruction attempts, such as reconstructed energy and distance from the primary vertex.

Light pattern Variables in this category attempt to determine whether the hits are geometrically consistent with a Cherenkov cone, which, for instance, should have an opening angle of $42^{\circ}$ and suffer exponential attenuation in water according to characteristic length $\mathrm{L}_{\text {att }}$. Examples include the reconstructed opening angle $\theta_{C}$, a measure of azimuthal symmetry, $\phi_{R M S}$, and the number of "backward" hits with respect with the Cherenkov cone, $\mathrm{N}_{\mathrm{BACK}}$.

Noise identification These variables exploit distinctive features of specific noise categories, such as highly-clustered flasher events, or highly charged hits from misbehaving PMTs.

The 22 observables are then used as features to train the BDT. After training, the BDT can be applied to neutron candidates and yield a single output ranging from 0 to 1 , which can be cut on to discriminate real neutrons from accidentals. Depending on the energy range, final signal efficiencies of $\sim 18-30 \%$ are achieved, with a background rate of 0.2-3 accidental coincidences/event.

\subsection{Systematic uncertainties}

Background rate uncertainty: Systematic uncertainty in the background efficiency of the neutron tagging cut arises due to the evolution of the detector's properties over time. Since the algorithm is sensitive to changes in the low-energy background signatures at SK, the time-dependence of the dark noise over the SK-IV period, in large part caused by a gradual PMT gain increase, affects the background efficiency of the BDT. In order to evaluate this effect, we separate the background data over all the SK-IV period into 10 time bins of about eight months each, and evaluate the maximal discrepancy for a range of BDT cuts. 
Signal efficiency uncertainty: Systematic uncertainty on neutron tagging efficiency stems from mismodeling of the $2.2 \mathrm{MeV}$ neutron capture signal in the MC samples used for the BDT training. To estimate the magnitude of the uncertainty, we use data samples containing real neutron captures from SK calibration runs. During calibration, a source containing Americium-241 and Beryllium9, embedded in a Bismuth-Germanium oxide (BGO) scintillator is placed in the detector. The source produces pairs of prompt and delayed neutron capture signals, mimicking the DSNB $\bar{v}_{e}$ signal. The source is placed at three different locations inside the tank and data runs from 2009 and 2016 are used, allowing to account for variation in vertex location and time. Thanks to the amplification efffect of the scintillator, the prompt event is readily identifiable after a cut on the total photoelectrons and other quality cuts. The neutron tagging BDT is then applied to the remaining events. The large number of neutron captures in the calibration runs allows us to extract the BDT efficiency on data in a statistical manner. The neutron capture time follows a decaying exponential distribution with $\left(\tau_{\text {cap }}=204.8 \mu \mathrm{s}\right)$ starting from the prompt event time, while uncorrelated backgrounds are flat in time by definition. The efficiency of the procedure is then extracted by fitting a decaying exponential with a constant term to the timing of neutron candidates passing the BDT cut. The efficiency on calibration data is compared to the efficiency in MC simulated with fixed IBD vertices corresponding to the source location in data, and injected with background data taken during calibration. The data and MC efficiency are compared across periods and tank locations, and an overall uncertainty of $7 \%$ is assigned.
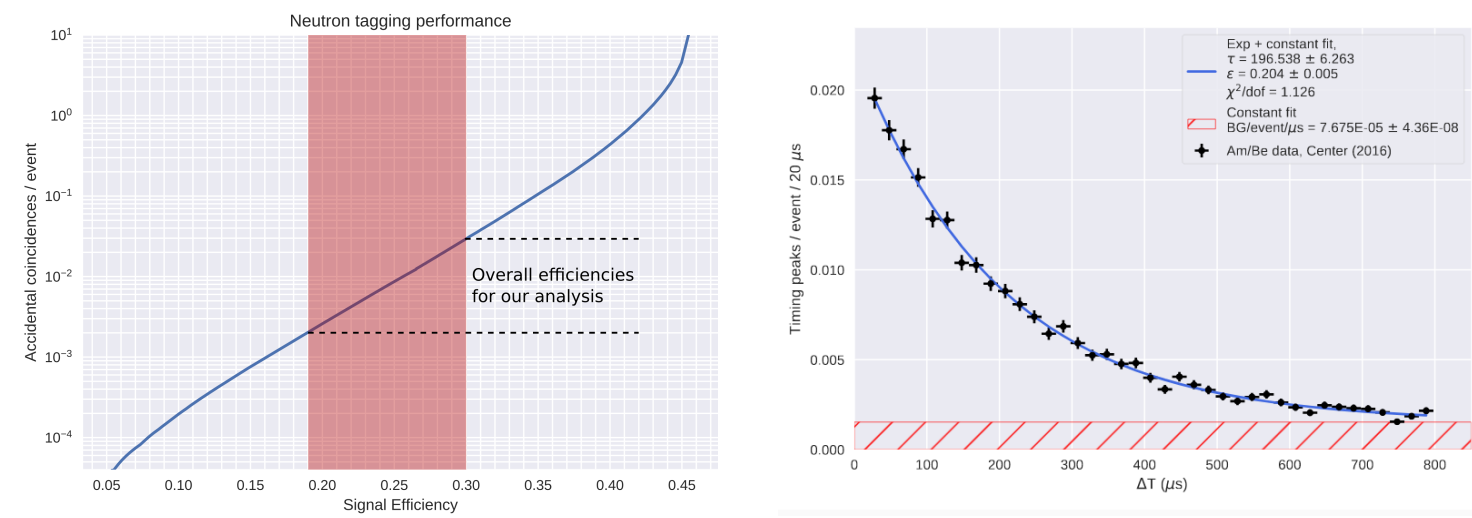

Figure 1: Left: number of neutron candidates from accidental background/event as function of signal efficiency for a range of neutron tagging BDT cuts. Right: exponential + constant fit of neutron candidate capture time in calibration data, after application of a neutron tagging BDT cut.

\section{Spectral analysis with SK-IV using neutron tagging}

In order to derive model-dependent upper limits on the DSNB flux, we carry out fits on the SKIV data after reduction cuts, using the spectral shapes from theoretical DSNB models and expected irreducible background contributions, extending the fitting method used for DSNB searches with SK-I-II-III [5]. Other than the neutron tagging cut, described in Section 2, these reduction cuts are chiefly aimed at characterizing the positron and removing comic muon spallation. 
This analysis focuses on the $16-80 \mathrm{MeV}$ region, where atmospheric neutrino backgrounds are expected to dominate after cuts. To evaluate the number of atmospheric background events we define three regions of parameter space based on the value of the Cherenkov angle: one signal region with $\theta_{C} \in[38,50]^{\circ}$ degrees that will contain most of the signal and the background events involving final-state electrons, and two sidebands for $\theta_{C} \in[20,38]^{\circ}$ and $\theta_{C} \in[78,90]^{\circ}$ respectively. The low Cherenkov angle region will be populated with mostly atmospheric backgrounds involving visible muons and pions while the high angle region will be mostly populated by $\mathrm{NC}$ atmospheric events with multiple final-state photons. In addition, we use the neutron tagging BDT cut to further separate events with exactly one identified neutron (that are therefore IBD-like) from the others. Due to the low efficiencies of the neutron tagging cuts, the non-IBD-like region is expected to contain a sizable amount of signal. Our analysis will then involve six regions of parameter space, as summarized in table 1. This procedure allows us to exploit neutron tagging whenever possible, since the 1-neutron signal region is a cleaner fitting region, with less contamination from atmospheric backgrounds, that will this be much more sensitive to the DSNB signal. At the same time, we still analyze candidates that fail the BDT cut.

\begin{tabular}{|cc|ccc|}
\hline & Cherenkov angle & $20-38^{\circ}$ & $38-50^{\circ}$ & $78-90^{\circ}$ \\
\hline Neutrons & & $\mu / \pi$ & Signal & NC \\
& 1 & $\mu / \pi$ & Signal & NC \\
\hline
\end{tabular}

Table 1: Overview of the regions used in this analysis. We divide the parameter space according to the Cherenkov angle and the number of tagged neutrons.

\subsection{DSNB Spectral shape fitting}

We perform a simultaneous fitting of the signal and background spectra to the data in all six regions of parameter space defined in table 1 using an unbinned extended maximum likelihood analysis. Here, we divide the atmospheric backgrounds into four categories with complementary spectral shapes, that we define as follows:

- Decay electrons: events with electrons from the decays of invisible muons and pions.

- CC $v_{e}$ : CC interactions of $v_{e}$ and $\bar{v}_{e}$, with no visible muons and pions in the final state.

- $\mu / \pi$ : CC interactions with visible muons and pions

- NC: all NC interactions

The decay electron spectral shape, following a well-known Michel spectrum, can be obtained with negligible associated systematic uncertainty directly from cosmic muon samples in data. All other background categories are obtained from MC simulation of atmospheric neutrinos. For each background category we define PDFs spanning all six Cherenkov angle and neutron regions. These PDFs are normalized to 1 across all regions. Similarly, for a given DSNB model, we define the reconstructed signal spectrum in each Cherenkov and neutron region by reweighting the IBD MC 
simulation. For $N_{\text {events }}$ observed events with energies $\left\{E_{1}, \ldots, E_{N}\right\}$ we can then extract the numbers of signal and background events by maximizing the following likelihood:

$$
\mathcal{L}\left(\left\{N_{j}\right\}\right)=e^{-\sum_{j=1}^{5} N_{j}} \prod_{i=1}^{N_{\text {events }}} \sum_{j=1}^{5} N_{j} \operatorname{PDF}_{j}\left(E_{i} ;, \theta_{c, i}, N_{i}^{\text {neutron }}\right)
$$

where $\left\{N_{j}\right\}$ are the numbers of events in the four background categories and for the signal, across all six signal and sideband regions. For each event, $\operatorname{PDF}_{j}\left(E_{i} ;, \theta_{c, i}, N_{i}^{\text {neutron }}\right)$ is the PDF for the signal or background category $j$ and in the region corresponding to the Cherenkov angle and the number of tagged neutrons of event $i$.

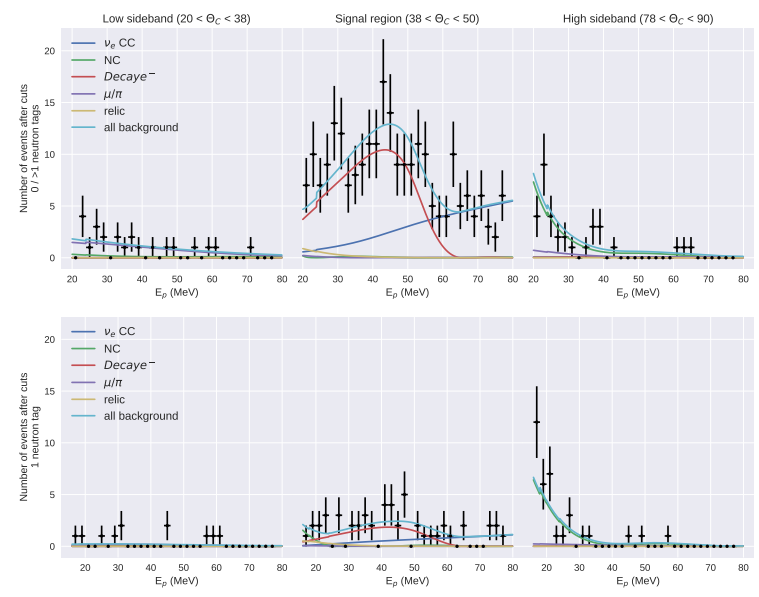

Figure 2: SK-IV spectral shape fit of the Large Mixing Angle DSNB model and expected background contributions from atmospheric neutrino backgrounds for the six regions of parameters space described in Table 1. Top row: 0/>1 tagged neutrons. Bottom row: exactly 1 tagged neutron. Middle: signal Cherenkov angle region. Left, right: Cherenkov angle sidebands.

\subsection{Systematic uncertainties:}

We incorporate systematic uncertainties into our study as nuisance parameters to the likelihood function in equation 1. Large uncertainties due to possible mismodeling of background contributions, of $O(10 \%)$, are included as distortions of the nominal PDFs. In particular, inclusion of neutron tagging in the analysis introduces systematic uncertainty related to mismodeling of the number of secondary neutrons in the final state of atmospheric neutrino interactions, affecting how each background is distributed across the two neutron regions. Each systematic uncertainty $k$ is parametrized as a distortion of the nominal background PDFs according to a single parameter $\alpha_{k}$. The likelihood is then convolved with a Bayesian prior $\pi_{k}\left(\alpha_{k}\right)$ :

$$
\mathcal{L}^{\prime}=\int \mathcal{L}\left(\left\{\alpha_{k}\right\}\right) \prod_{k} \pi_{k}\left(\alpha_{k}\right) \mathrm{d} \alpha_{k}
$$

\subsection{Results}

We apply the fitting procedure to the data collected during SK-IV for various models, and combine the results with fits on data from previous periods (SK-I-II-III). The SK-IV fit for the 
Large Mixing Angle model [6, 7] is shown in Figure 2 Background rates are particularly low in the one-neutron region for the SK-IV analysis, which makes it particularly sensitive to IBD signals in spite of the low efficiency of the neutron tagging cut. In future analyses with $\mathrm{Gd}$, increases in neutron tagging efficiency will make this region the main signal region, affording higher sensitivity to the DSNB. While a downward fluctuation, leading to particularly strong limits, is observed in SK-I, in all other phases of SK we observe a slight excess of around $2 \sigma$. Due to the slight statistical excess observed, many optimistic models aren't excluded yet; however, the combined sensitivity of SK-I,II,III,IV at $90 \%$ confidence level is comparable to their predicted fluxes, and is currently the best sensitivity to the DSNB among neutrino observatories.

\section{Sensitivity projections of current method with Gd}

We make preliminary projections of the sensitivity of SK to different DSNB models with Gddoped water. So far, we have made projections for an analysis closely following the procedure of the current search. Using SK detector simulations of neutron captures in Gd-doped water, we train a new BDT using the same observables as described in Section 2.2. Here, nearly all neutron captures by Gd pass the candidate selection stage, while also being easier to separate from background by the BDT thanks to the higher total energy of the photon cascade. For a nominal Gd concentration of $0.1 \%$, in which $\sim 90 \%$ of the neutrons produced will be captured by $\mathrm{Gd}$, the overall neutron tagging efficiency is estimated at $\sim 80 \%$, requiring a similar background rejection for the current analysis in pure water, an efficiency improvement of up to 4 times. To project the impact of the improved efficiency on the analysis, we generate $O\left(10^{4}\right)$ pseudo-experiments starting from the expected background distributions, distorted according to the associated systematic uncertainties, and study the resulting limits on the DSNB flux. The 90\% C.L. sensitivity for a 10 years of SK livetime at nominal Gd concentration obtained is roughly $20 \%$ better than the $\sim 20$-year SKI-II-III-IV combined runs considered by the current analysis. With such a 10 -year run, before any combination with other SK runs, we would reach $2 \sigma$ sensitivity to optimistic DSNB models. Further gains in sensitivity are expected, for example with improved rejection of neutron-producing backgrounds that may pass neutron tagging cuts.

\section{References}

[1] J.F. Beacom, The Diffuse Supernova Neutrino Background, Ann. Rev. Nucl. Part. Sci. 60 (2010) 439 [1004 . 3311$].$

[2] C.W. Walter, The Super-Kamiokande Experiment, 0802 . 1041.

[3] C.X. and, Current status of SK-gd project and EGADS, Journal of Physics: Conference Series 718 (2016) 062070.

[4] S. Yamada, K. Awai, Y. Hayato, K. Kaneyuki, Y. Kouzuma, S. Nakayama et al., Commissioning of the new electronics and online system for the super-kamiokande experiment, IEEE Transactions on Nuclear Science 57 (2010) 428.

[5] Super-Kamiokande collaboration, Supernova Relic Neutrino Search at Super-Kamiokande, Phys. Rev. D 85 (2012) 052007 [1111.5031].

[6] S. Ando, K. Sato and T. Totani, Detectability of the supernova relic neutrinos, Nuclear Physics A 721 (2003) C541.

[7] The LMA model flux has been adjusted, increasing by a factor of 2.56 from the original paper, at NNN05. 


\section{Full Authors List: Super-Kamiokande Collaboration}

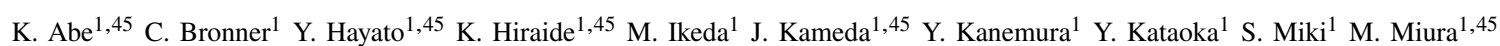
S. Moriyama ${ }^{1,45}$ Y. Nagao ${ }^{1}$ M. Nakahata ${ }^{1,45}$ S. Nakayama ${ }^{1,45}$ K. Okamoto ${ }^{1}$ G. Pronost ${ }^{1}$ H. Sekiya ${ }^{1,45}$ M. Shiozawa ${ }^{1,45}$ Y. Sonoda $^{1}$ Y. Suzuki ${ }^{1}$ A. Takeda ${ }^{1,45}$ Y. Takemoto ${ }^{1}$ A. Takenaka ${ }^{1}$ H. Tanaka ${ }^{1}$ S. Watanabe ${ }^{1}$ T. Yano ${ }^{1}$ S. Han ${ }^{2}$ T. Kajita ${ }^{2}, 45$ K. Okumura ${ }^{2,45}$ T. Tashiro ${ }^{2}$ J. Xia ${ }^{2}$ G. D. Megias ${ }^{3}$ L. Labarga ${ }^{4}$ Ll. Marti ${ }^{4}$ B. Zaldivar ${ }^{4}$ B. W. Pointon ${ }^{6,49}$ E. Kearns ${ }^{5,45}$ J. L. Raaf ${ }^{5}$ L. Wan ${ }^{5}$ T. Wester ${ }^{5}$

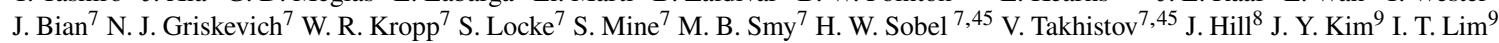
R. G. Park ${ }^{9}$ B. Bodur ${ }^{10}$ K. Scholberg ${ }^{10}$ C. W. Walter ${ }^{10,45}$ L. Bernard ${ }^{11}$ A. Coffani ${ }^{11}$ O. Drapier ${ }^{11}$ S. El Hedri ${ }^{11}$ A. Giampaolo ${ }^{11}$ M. Gonin ${ }^{11}$ Th. A. Mueller ${ }^{11}$ P. Paganini ${ }^{11}$ B. Quilain ${ }^{11}$ T. Ishizuka ${ }^{12}$ T. Nakamura ${ }^{13}$ J. S. Jang ${ }^{14}$ J. G. Learned ${ }^{15}$ S. Cao ${ }^{16}$ L. H. V. Anthony ${ }^{17}$ D. Martin ${ }^{17}$ M. Scott ${ }^{17}$ A. A. Sztuc ${ }^{17}$ Y. Uchida ${ }^{17}$ V. Berardi ${ }^{18}$ M. G. Catanesi ${ }^{18}$ E. Radicioni ${ }^{18}$ N. F. Calabria ${ }^{19}$ L. N. Machado ${ }^{19}$ G. De Rosa ${ }^{19}$ G. Collazuol ${ }^{20}$ F. Iacob $^{20}$ M. Lamoureux ${ }^{20}$ M. Mattiazzi ${ }^{20}$ N. Ospina ${ }^{20}$ L. Ludovici ${ }^{21}$ Y. Maekawa $^{22}$ Y. Nishimura ${ }^{22}$ M. Friend ${ }^{23}$ T. Hasegawa ${ }^{23}$ T. Ishida ${ }^{23}$ T. Kobayashi ${ }^{23}$ M. Jakkapu ${ }^{23}$ T. Matsubara ${ }^{23}$ T. Nakadaira ${ }^{23}$ K. Nakamura ${ }^{23,45}$ Y. Oyama ${ }^{23}$ K. Sakashita ${ }^{23}$ T. Sekiguchi ${ }^{23}$ T. Tsukamoto 23 T. Boschi ${ }^{24}$ F. Di Lodovico ${ }^{24}$ J. Gao $^{24}$ J. Migenda $^{24}$ M. Taani ${ }^{24}$ S. Zsoldos ${ }^{24}$ Y. Nakano ${ }^{25}$ H. Ozaki ${ }^{25}$ A. T. Suzuki ${ }^{25}$ Y. Takeuchi ${ }^{25,45}$ S. Yamamoto ${ }^{25}$ Y. Kotsar ${ }^{25}$ A. Ali ${ }^{26}$ J. Feng $^{26}$ T. Kikawa ${ }^{26}$ M. Mori $^{26}$ T. Nakaya $a^{26,45}$ R. A. Wendell ${ }^{26,45}$ K. Yasutome ${ }^{26}$ P. Fernandez ${ }^{28}$ N. McCauley ${ }^{28}$ P. Mehta ${ }^{28}$ K. M. Tsui ${ }^{27}$ Y. Fukuda ${ }^{28}$ Y. Itow ${ }^{29,30}$ H. Menjo ${ }^{29}$ K. Sato ${ }^{29}$ J. Lagoda ${ }^{31}$ S. M. Lakshmi ${ }^{31}$ P. Mijakowski ${ }^{31}$ J. Zalipska ${ }^{31}$ J. Jiang ${ }^{32}$ C. K. Jung ${ }^{32}$ C. Vilela ${ }^{32}$ M. J. Wilking ${ }^{32}$

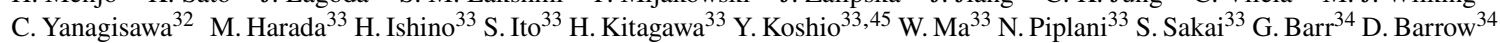
L. Cook ${ }^{34,45}$ A. Goldsack ${ }^{34,45}$ S. Samani ${ }^{34}$ D. Wark ${ }^{34,39}$ F. Nova ${ }^{35}$ J. Y. Yang ${ }^{36}$ S. J. Jenkins ${ }^{37}$ M. Malek ${ }^{37}$ J. M. McElwee ${ }^{37}$ O. Stone ${ }^{37}$

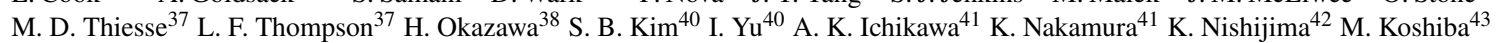
K. Iwamoto ${ }^{44}$ Y. Nakajima ${ }^{44}$ N. Ogawa ${ }^{44}$ M. Yokoyama ${ }^{44,45}$ K. Martens ${ }^{45}$ M. R. Vagins ${ }^{45,7}$ M. Kuze ${ }^{46}$ S. Izumiyama ${ }^{46}$ M. Inomoto $^{47}$ M. Ishitsuka $a^{47}$ H. Ito ${ }^{47}$ T. Kinoshita ${ }^{47}$ R. Matsumoto ${ }^{47}$ M. Shinoki ${ }^{47}$ T. Suganuma ${ }^{47}$ J. F. Martin ${ }^{48}$ H. A. Tanaka ${ }^{48}$ T. Towstego $0^{48}$ R. Akutsu ${ }^{49}$ P. de Perio ${ }^{49}$ M. Hartz ${ }^{49}$ A. Konaka ${ }^{49}$ N. W. Prouse ${ }^{49}$ S. Chen ${ }^{50}$ B. D. Xu ${ }^{50}$ M. Posiadala-Zezula ${ }^{51}$ D. Hadley ${ }^{52}$

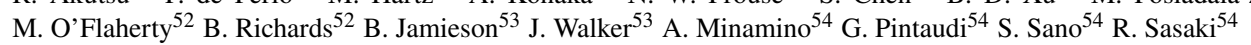

${ }^{1}$ Kamioka Observatory, Institute for Cosmic Ray Research, University of Tokyo, Kamioka, Gifu 506-1205, Japan.

${ }^{2}$ Research Center for Cosmic Neutrinos, Institute for Cosmic Ray Research, University of Tokyo, Kashiwa, Chiba 277-8582, Japan.

${ }^{3}$ Institute for Cosmic Ray Research, University of Tokyo, Kashiwa, Chiba 277-8582, Japan.
${ }^{4}$ Department of Theoretical Physics, University Autonoma Madrid, 28049 Madrid, Spain.

${ }^{5}$ Department of Theoretical Physics, University Autonoma Madrid, 28049

${ }^{6}$ Department of Physics, British Columbia Institute of Technology, Burnaby, BC, V5G 3H2, Canada.

${ }^{7}$ Department of Physics and Astronomy, University of California, Irvine, Irvine, CA 92697-4575, USA

${ }^{8}$ Department of Physics, California State University, Dominguez Hills, Carson, CA 90747, USA.

${ }^{9}$ Institute for Universe and Elementary Particles, Chonnam National University, Gwangju 61186, Korea

${ }^{10}$ Department of Physics, Duke University, Durham NC 27708, USA

${ }^{11}$ Ecole Polytechnique, IN2P3-CNRS, Laboratoire Leprince-Ringuet, F-91120 Palaiseau, France.

${ }_{12}^{12}$ Junior College, Fukuoka Institute of Technology, Fukuoka, Fukuoka 811-0295, Japan.

${ }^{13}$ Department of Physics, Gifu University, Gifu, Gifu 501-1193, Japan.

${ }^{14}$ GIST College, Gwangju Institute of Science and Technology, Gwangju 500-712, Korea.

${ }^{15}$ Department of Physics and Astronomy, University of Hawaii, Honolulu, HI 96822, USA

${ }^{16}$ Institute For Interdisciplinary Research in Science and Education, ICISE, Quy Nhon, 55121, Vietnam
.

${ }^{17}$ Department of Physics, Imperial College London, London, SW7 2AZ, United Kingdom

${ }^{18}$ Dipartimento Interuniversitario di Fisica, INFN Sezione di Bari and Università e Politecnico di Bari, I-70125, Bari, Italy.

${ }^{19}$ Dipartimento di Fisica, INFN Sezione di Napoli and Università di Napoli, I-80126, Napoli, Italy.

${ }^{20}$ Dipartimento di Fisica, INFN Sezione di Padova and Università di Padova, I-35131, Padova, Italy.

${ }^{21}$ INFN Sezione di Roma and Università di Roma "La Sapienza", I-00185, Roma, Italy.

${ }^{22}$ Department of Physics, Keio University, Yokohama, Kanagawa, 223-8522, Japan.

${ }^{23}$ High Energy Accelerator Research Organization (KEK), Tsukuba, Ibaraki 305-0801, Japan

${ }^{24}$ Department of Physics, King's College London, London, WC2R 2LS, UK.

${ }^{25}$ Department of Physics, Kobe University, Kobe, Hyogo 657-8501, Japan.

${ }^{26}$ Department of Physics, Kyoto University, Kyoto, Kyoto 606-8502, Japan.

${ }^{27}$ Department of Physics, University of Liverpool, Liverpool, L69 7ZE, United Kingdom.

${ }^{28}$ Department of Physics, Miyagi University of Education, Sendai, Miyagi 980-0845, Japan.

${ }^{29}$ Institute for Space-Earth Environmental Research, Nagoya University, Nagoya, Aichi 464-8602, Japan.

${ }^{30}$ Kobayashi-Maskawa Institute for the Origin of Particles and the Universe, Nagoya University, Nagoya, Aichi 464-8602, Japan.

${ }^{31}$ National Centre For Nuclear Research, 02-093 Warsaw, Poland.

${ }^{32}$ Department of Physics and Astronomy, State University of New York at Stony Brook, NY 11794-3800, USA

${ }^{33}$ Department of Physics, Okayama University, Okayama, Okayama 700-8530, Japan

${ }^{34}$ Department of Physics, Oxford University, Oxford, OX1 3PU, United Kingdom.

${ }_{36}^{35}$ Rutherford Appleton Laboratory, Harwell, Oxford, OX11 0QX, UK.

${ }^{36}$ Department of Physics, Seoul National University, Seoul 151-742, Korea.

${ }^{37}$ Department of Physics and Astronomy, University of Sheffield, S3 7RH, Sheffield, United Kingdom.

${ }^{38}$ Department of Informatics in Social Welfare, Shizuoka University of Welfare, Yaizu, Shizuoka, 425-8611, Japan.

${ }^{39}$ STFC, Rutherford Appleton Laboratory, Harwell Oxford, and Daresbury Laboratory, Warrington, OX11 0QX, United Kingdom.

${ }^{40}$ Department of Physics, Sungkyunkwan University, Suwon 440-746, Korea.

${ }^{41}$ Department of Physics, Faculty of Science, Tohoku University, Sendai, Miyagi, 980-8578, Japan.

${ }^{42}$ Department of Physics, Tokai University, Hiratsuka, Kanagawa 259-1292, Japan.

${ }^{43}$ The University of Tokyo, Bunkyo, Tokyo 113-0033, Japan.

${ }^{44}$ Department of Physics, University of Tokyo, Bunkyo, Tokyo 113-0033, Japan.

${ }^{45}$ Kavli Institute for the Physics and Mathematics of the Universe (WPI), The University of Tokyo Institutes for Advanced Study, University of Tokyo, Kashiwa, Chiba 277-8583, Japan.

${ }^{46}$ Department of Physics, Tokyo Institute of Technology, Meguro, Tokyo 152-8551, Japan.

${ }_{48}^{47}$ Department of Physics, Faculty of Science and Technology, Tokyo University of Science, Noda, Chiba 278-8510, Japan

${ }^{48}$ Department of Physics, University of Toronto, ON, M5S 1A7, Canada.

${ }^{49}$ TRIUMF, 4004 Wesbrook Mall, Vancouver, BC, V6T2A3, Canada.

${ }^{50}$ Department of Engineering Physics, Tsinghua University, Beijing, 100084, China.

${ }^{51}$ Faculty of Physics, University of Warsaw, Warsaw, 02-093, Poland.

${ }^{52}$ Department of Physics, University of Warwick, Coventry, CV4 7AL, UK.

${ }^{53}$ Department of Physics, University of Winnipeg, MB R3J 3L8, Canada.

${ }^{54}$ Department of Physics, Yokohama National University, Yokohama, Kanagawa, 240-8501, Japan.

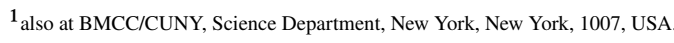

${ }^{2}$ Deceased.
} 